\title{
Lightweight Deep Learning for Malaria Parasite Detection Using Cell-Image of Blood Smear Images
}

\author{
Amin Alqudah ${ }^{1}$, Ali Mohammad Alqudah ${ }^{2 *}$, Shoroq Qazan ${ }^{1}$ \\ ${ }^{1}$ Department of Computer Engineering, Hijjawi Faculty for Engineering Technology, Yarmouk University, Shafiq Irshidat \\ Street, Irbid 21163, Jordan \\ ${ }^{2}$ Department of Biomedical Systems and Informatics Engineering, Hijjawi Faculty for Engineering Technology, Yarmouk \\ University, Shafiq Irshidat Street, Irbid 21163, Jordan
}

Corresponding Author Email: ali_qudah@yu.edu.jo

https://doi.org/10.18280/ria.340506

Received: 26 July 2020

Accepted: 10 October 2020

\section{Keywords:}

deep learning, convolutional neural networks, malaria, classification, computeraided diagnosis, blood smear

\begin{abstract}
Malaria is an infectious disease that is caused by the plasmodium parasite which is a singlecelled group. This disease is usually spread employing an infected female anopheles mosquito. Recent statistics show that in 2017 there were only around 219 million recorded cases and about 435,000 deaths were reported due to this disease and more than $40 \%$ of the global population is at risk. Despite this, many image processing fused with machine learning algorithms were developed by researchers for the early detection of malaria using blood smear images. This research used a new CNN model using transfer learning for classifying segmented infected and Uninfected red blood cells. The experimental results show that the proposed architecture success to detect malaria with an accuracy of $98.85 \%$, sensitivity of $98.79 \%$, and a specificity of $98.90 \%$ with the highest speed and smallest input size among all previously used CNN models.
\end{abstract}

\section{INTRODUCTION}

Malaria is classified as a contagious disease which is caused by a single-celled microorganism which is belonging to the genus protozoan parasite of the plasmodium group where five of their species can infect humans. The disease is mainly spread by the mean of bite infected female Anopheles Mosquito. Based on recent statistics the malaria disease puts around $40 \%$ of the world population to risk with nearly 240 million cases reported each year, African and especially the Sub-Saharan-Africa countries are the most due to malaria [1]. Figure 1 shows worldwide malaria new cases rates per 1,000 population.

World Health Organization (WHO) reports show that around $90 \%$ of the worldwide malaria infections and death cases happened in Africa were kids under 5 years are representing the highest percentage of the malaria-infected deaths $[1,2]$. In general, usual malaria signs and indications are fever, nausea, and headaches. In some acute and severe cases, patients may have yellow skin, seizures, and coma which may lead to death. Every year, well-trained experts at hospitals around the world examined millions of blood films in order to detect malaria cases. The detection of malaria cases usually requires a manual process especially in counting the parasites and infected red blood cells, which is considered time-consuming and may cause some errors [3, 4].

Generally, there are two main scientific approaches commonly used to diagnose malaria: microscopy of thin blood cells and an antigen diagnostic examination. The former is a very time-consuming operation, typically with doctors a minimum of 5000 cells must be identified manually to validate, the condition, although the latter is much quicker than the former one, the antigen-based fast diagnostic tests are less effective. In addition, malaria is typically distributed across poverty and instability. In areas with poor psychiatric outcomes, patients cannot be admitted timely care or offer reimbursement for antigen-based accelerated treatment diagnostic research. Finding a book, effective, is very meaningful and a convenient method for diagnosing malaria $[1,3,4]$.

However, this process is completely depending on the skill of the microscopist, and working in an environment with limited resource set-up and without helpful systems for improvement of the skills of microscopist or computer-aided diagnosis (CAD) systems will lead to affect the diagnostic quality and finally results to wrong diagnostic decisions, such systems can help and boost microscopist to enhance the accuracy of blood films classification and malaria detection [3-6].

In recent years, several classification experiments have been performed on automated malaria-infected cells in medical pictures, such as approaches focused on machine learning and morphology [4]. However, these approaches usually have drawbacks, such as having a lot of advanced expertise and generally require the help of a trained and skilled surgeon [5].

Recently, with the development of artificial intelligence (AI) based systems which is known as computer-aided diagnosis or decision support systems malaria detection using blood films became more efficient $[3,4]$. One of the most recent AI techniques is Deep learning (DL) which can be used to classify cell images and help to prevent wrong diagnostic decisions [5].

Deep learning is a sub-area of machine learning (ML) which gives exceptional performance in different medical fields. This is because DL deals with raw and multidimensional data (1D or 2D). The applications of deep learning are not limited to the 
medical fields; in recent years deep learning pays the attention of researchers, and its applications grew exponentially [6]. In recent times, many artificial intelligence techniques have been applied for detecting malaria based on blood film images [57]. Examples of these techniques are artificial neural networks (ANN's), support vector machine (SVM), and convolutional neural networks (CNN's).

$\mathrm{CNN}$ is the most recent technological development and modern technique in the field of DL, and it is used widely in the field of computer vision for the diagnosis of medical disease and especially for data that are based on images. CNN's have started to be used as a necessary approach to classify and diagnose medical images. They do not require any feature extraction before the training process $[7,8]$. In general, CNN's are designed to minimize or eliminate data preprocessing steps. They are compatible to deal with raw images or data $[9,10]$.

This paper proposed a transfer learning of AOCT-NET CNN model for malaria detection using Red Blood Cells (RBCs), this research paper focuses on providing a new transfer learning $\mathrm{CNN}$ architecture rather than using the traditional transfer learning models (AlexNet, GoogleNet, VGG16, et al.), a method of deep learning, for malaria cellimage classification. Our main contributions can be summarized as follows:

(1) We used a new design, lightweight, and very efficient network architecture.

(2) We train and evaluate our proposed design using different scenarios.

Compare it with several well-established models. And the results prove that we have achieved state-of-art performance on malaria-infected red blood cell classification.

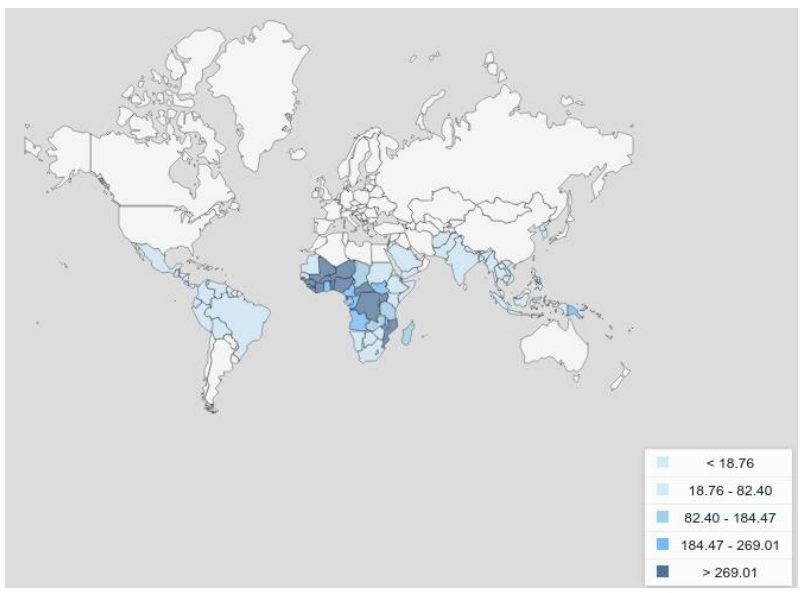

Figure 1. Worldwide malaria new cases rates per 1,000 population

\section{MATERIALS AND METHODS}

In this section, the used dataset of red blood cells (RBCs) and the methodology including the convolutional neural network $(\mathrm{CNN})$ architecture and training process are discussed in detail. Figure 2 shows the flow diagram or a schematic of the proposed methodology.

\subsection{The dataset}

The dataset used in the implementation contains 27,558 images of segmented red blood cells (RBCs) for both parasitized and uninfected cells and appeared for the first time [3]. The segmentation process was developed and applied on the dataset by Rajaraman et al. and can be found by Rajaraman et al. [4]. The Dataset is available online for free at the official website of the National Library of Medicine (NLM) at https://lhncbc.nlm.nih.gov/publication/pub9932. According to the dataset, the difference between malaria-infected and uninfected red blood cells (RBCs) can be noticed in Figure 3. Table 1 shows the total number of images for each class in the dataset. The images in the dataset we resized to a size of $64 \times 64$ pixels instead of size $127 \times 130$ pixels. This input size of the used CNN architecture and all input images must have the same size.

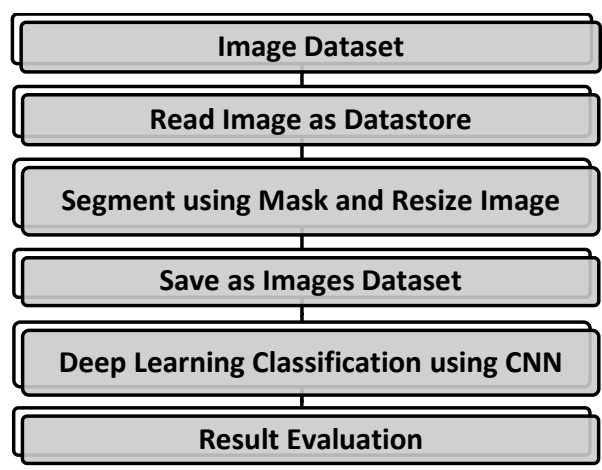

Figure 2. Flow diagram of the proposed methodology
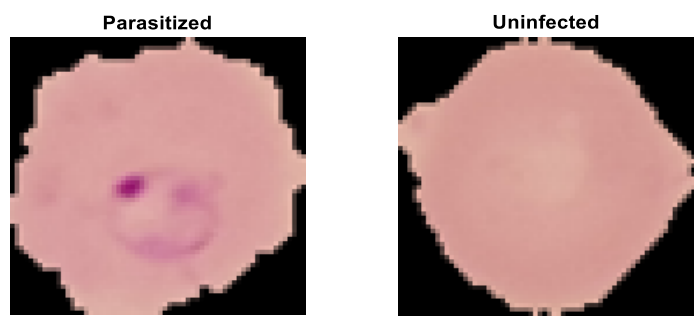

Figure 3. Sample Images of Infected and Uninfected Red Blood Cells (RBC)

Table 1. Summary of the used dataset

\begin{tabular}{ccccc}
\hline Class & $\begin{array}{c}\text { Training } \\
\text { Images }\end{array}$ & $\begin{array}{c}\text { Validation } \\
\text { Images }\end{array}$ & $\begin{array}{c}\text { Testing } \\
\text { Images }\end{array}$ & $\begin{array}{c}\text { Total } \\
\text { Images }\end{array}$ \\
\hline Parasitized & 9645 & 2067 & 2067 & 13779 \\
Uninfected & 9645 & 2067 & 2067 & 13779 \\
\hline
\end{tabular}

\subsection{Convolutional Neural Network (CNN)}

Artificial neural networks (ANNs) and their enhancement like Convolutional Neural Network (CNN) type are the top, most recent, and widely used deep neural networks (DNNs) that can treat different types of raw data either images or signals [9]. The CNNs are composed of specified stacked layers. The layers are mainly, input layer, convolution layer, rectified linear unit (ReLU) layer, fully connected layer, classification layer, and output layer [8]. Basically, $\mathrm{CNN}$ is based on two main processes namely, convolution and downsampling.

The convolution is done using a trainable and adaptive filter which has pre-specified size and weights that can be adjusted during the training phase and down-sampling [10]. In this research, the segmented red blood cells (RBCs) images are stored as an image datastore with two categories created, each 
one of these categories consists of images for a specific class of parasitized and uninfected RBCs. The datastore is divided into three sub-datastores: training, validation, and testing subdatastores. In the training stage, $70 \%$ of the data is utilized, $15 \%$ for validation, and the rest $(15 \%)$ is used in the test stage $[11,12]$. In this research, a new customized CNN architecture is investigated, and later we will explain the structure of the proposed $\mathrm{CNN}$ architecture.

In this paper, a new transferred learning $\mathrm{CNN}$ architecture is used which was proposed by Alqudah [11] and known as AOCT-NET. The architecture is mainly consisting of 18 layers that able to grade the RBCs into two classes effectively, the architecture is applied to the image datastore mentioned before and the performance of the architecture was monitored. Figure 4 shows the detailed structure of the used $\mathrm{CNN}$.

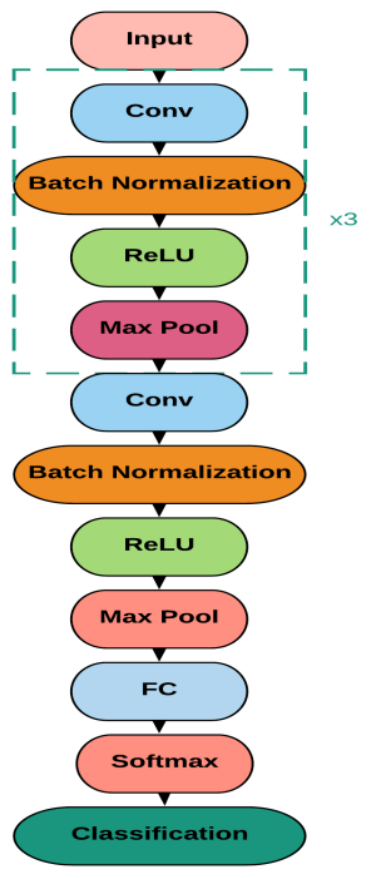

Figure 4. The used CNN architecture

\subsection{Performance evaluation}

To check the efficiency and the performance of the used customized $\mathrm{CNN}$ architecture in the classification of RBCs into parasitized and uninfected, a confusion matrix was generated [13]. In general, using the generated confusion matrix the following equations can be used to calculate the: accuracy, sensitivity, precision, and specificity, where these values measure how precisely the RBCs are classified [14]. Four statistical indices are used to measure the performance of the classification. These indices are true positive (TP), false positive (FP), false negative (FN), and true negative (TN). These four indices are calculated using the confusion matrix. The accuracy, sensitivity, and specificity, and precision were computed as [12-15]:

$$
\begin{gathered}
\text { Accuracy }=\frac{T P+T N}{T P+F P+T N+F N} \\
\text { Sensitivity }=\frac{T P}{T P+F N}
\end{gathered}
$$

$$
\begin{gathered}
\text { Specificity }=\frac{T N}{T N+F P} \\
\text { Precision }=\frac{T P}{T P+F P} \\
F 1-\text { Score }=2 * \frac{\text { Precision } * \text { Sensitivity }}{\text { recision }+ \text { Sensitivity }}
\end{gathered}
$$

\section{RESULTS}

To evaluate the proposed methodology performance, the methodology was implemented, and it was run using a desktop computer with Intel Core-I5 computer with $12 \mathrm{~Gb}$ of RAM. The images were tested with a minibatch size of 128 . The dataset was divided into three subsets: training, validation, and testing, with a percentage of $70 \%, 15 \%$, and $15 \%$, respectively.

To validate the best smallest size that we can use in building the CNN architecture we run the structure on different input size which is $64 \times 64,128 \times 128$, and $256 \times 256$. Table 2 shows the performance results of these input sizes. Based on this table we can conclude that $64 \times 64$ is the best input size by making a trade-off between the performance values and time.

Table 2. Performances of different input sizes on the used $\mathrm{CNN}$ architecture

\begin{tabular}{cccc}
\hline Input Size & $\mathbf{6 4 x 6 4}$ & $\mathbf{1 2 8 x 1 2 8}$ & $\mathbf{2 5 6 x 2 5 6}$ \\
\hline Accuracy & 98.85 & 98.90 & 98.90 \\
Sensitivity & 98.79 & 98.81 & 98.81 \\
Specificity & 98.90 & 98.91 & 98.91 \\
Precision & 98.90 & 98.91 & 98.91 \\
Time & 0.1352 & 0.2796 & 0.5421 \\
\hline
\end{tabular}

The optimization algorithm was adaptive moment estimation (Adam) with an initial learning rate of 0.001 which results in 15000 iterations. These values have been selected based on trying different scenarios of optimization algorithms with different values. Table 3 shows these scenarios and their results. Based on the results shown in Table 3 we can conclude that the best scenario was the ADAM with a 0.001 learning rate.

Table 3. Different optimization scenarios and the performances of the $\mathrm{CNN}$ architecture

\begin{tabular}{cccccc}
\hline & \multicolumn{3}{c}{ ADAM } & \multirow{2}{*}{ SGDM } & \multirow{2}{*}{ RMSPROP } \\
\cline { 2 - 4 } & $\mathbf{0 . 0 0 1}$ & $\mathbf{0 . 0 1}$ & $\mathbf{0 . 1}$ & & \\
\hline Accuracy & 98.85 & 98.80 & 98.51 & 97.95 & 95.31 \\
Sensitivity & 98.79 & 98.77 & 98.68 & 97.36 & 95.28 \\
Specificity & 98.90 & 98.83 & 98.77 & 97.31 & 94.87 \\
Precision & 98.90 & 98.83 & 98.77 & 97.31 & 94.87 \\
\hline
\end{tabular}

The following figures show the results of the proposed dataset with the designed CNN architecture. The accuracy and the loss of the training and validation data are shown in Figure 5. The testing confusion matrix and ROC are shown in Figure $6 \mathrm{~A}$ and $\mathrm{B}$, respectively. Based on these figures, it can be noticed that the proposed system has classified the images (red blood cells) with an accuracy rate of $98.85 \%$, sensitivity rate of $98.79 \%$, specificity rate of $98.90 \%$, and a precision rate of $98.90 \%$. 

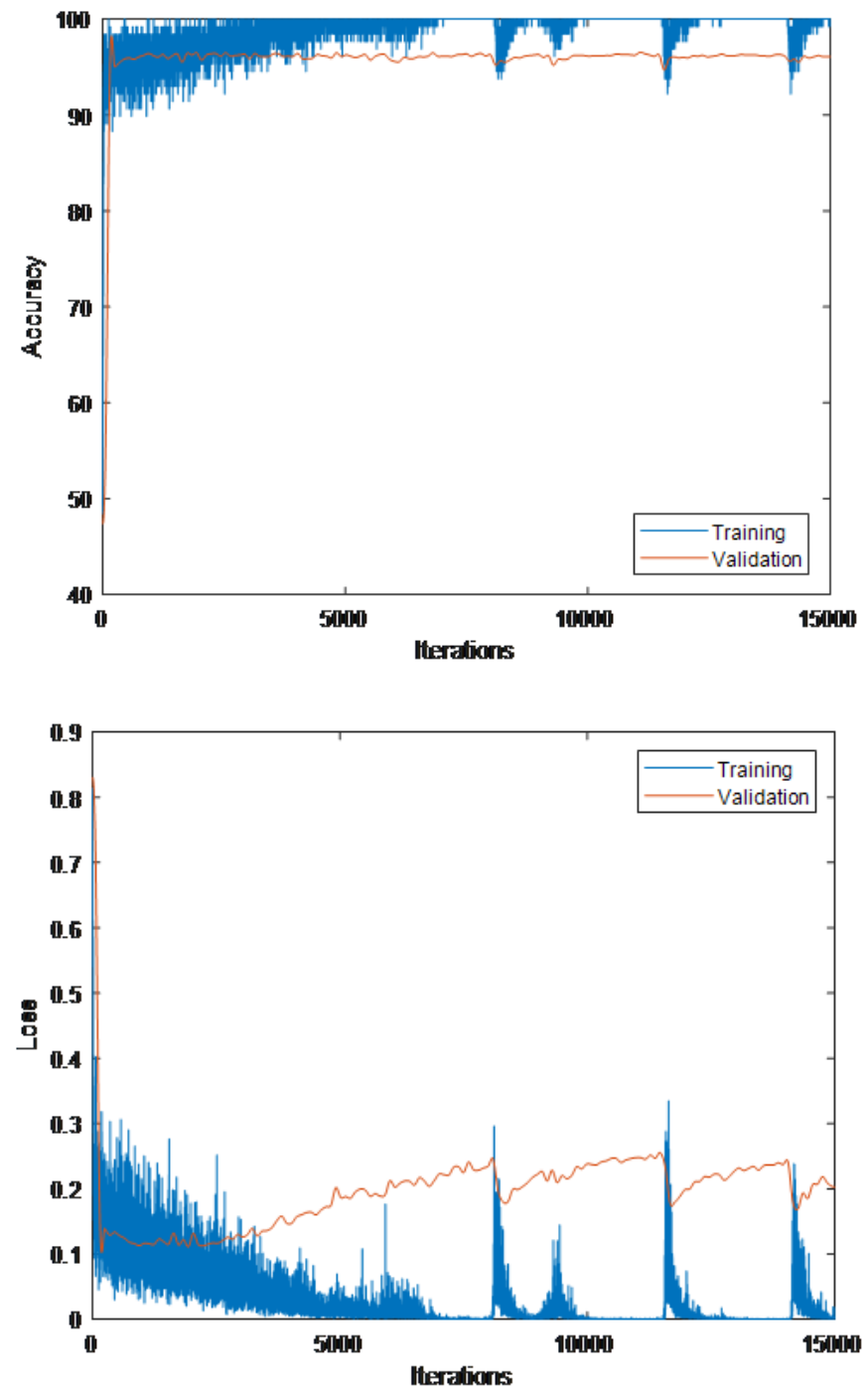

Figure 5. Upper: The training and validation accuracy; Lower: The training and validation loss

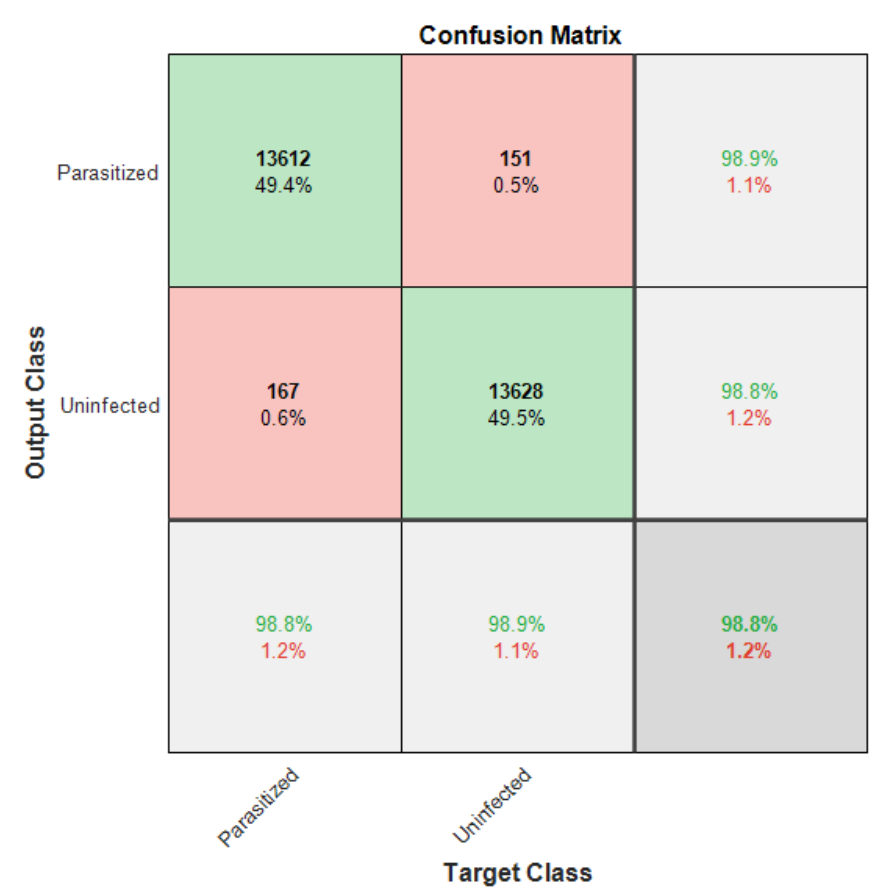

(A)

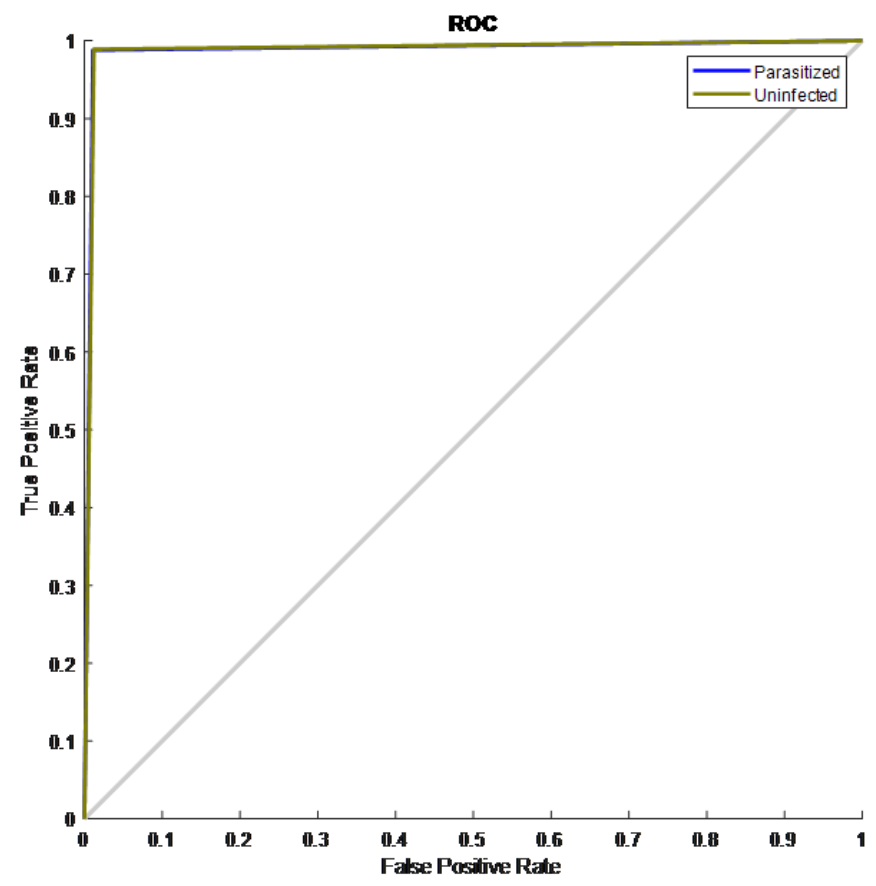

(B)

Figure 6. A: The Confusion Matrix; B: The ROC

We can also use the trained $\mathrm{CNN}$ for deep feature extractions from cell images. These deep features are automated, discriminated, and powerful features that can be used to build hybrid systems like CNN-SVM and CNN-KNN. Figure 7 below shows the extracted feature distribution.

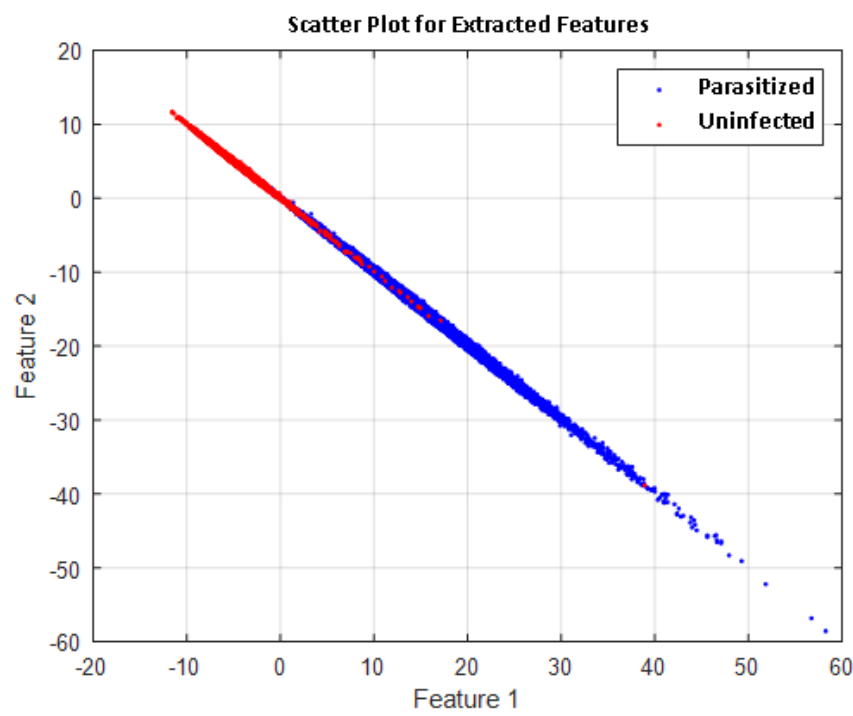

Figure 7. The extracted deep features using CNN

The proposed technique, using the CNN-based scheme for classifying cell images, proved to be effective in classifying high-performance colored images. Figure 8 displays the class activation mapping (CAM) for two cases: parasitized and uninfected using the last AOCT-Net ReLU layer.

In general, deep learning networks and models are considered and deal with them as "black boxes". This means that there is no way to determine out what a network has learned or which part of an input to the network was responsible for the prediction of the network. When these models malfunction and make inaccurate assumptions, they 
also fail spectacularly without any warning or clarification. Class activation mapping [16] is a method that can be used to visually illustrate the predictions of coevolutionary neural networks. Incorrect, seemingly unwise forecasts may also provide sound reasons. Using class activation mapping, you can verify whether a particular part of the input picture has "confused" the network to make an inaccurate assumption. Finally, CAM is a tool that is used to simulate where CNN looks and catches CNN's focus in the input image to retrieve the deep features [17]. Usage of Figure 8, we find that CNN architecture pays attention to places of concern cell has been affected (shown as a white spread area).

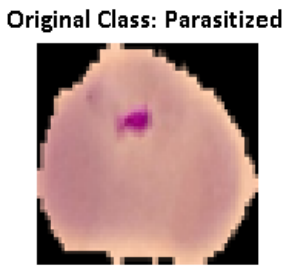

(a)

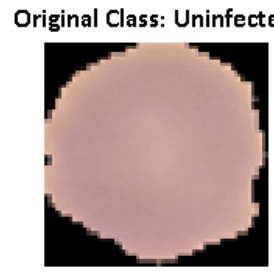

(d)

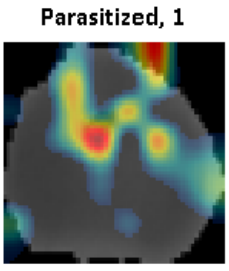

(b)

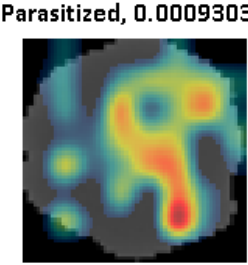

(e)

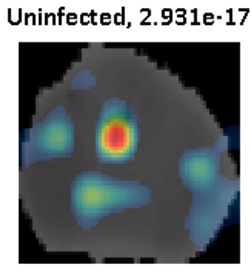

(c)

Uninfected, 0.99907

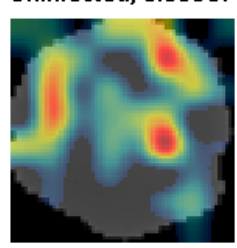

(f)
Figure 8. Class activation mapping for parasitized and uninfected: a) input image of parasitized class; b) parasitized features using CAM; c) uninfected features using CAM; d) input image of uninfected class; e) parasitized features using CAM; f) uninfected features using CAM

\section{DISCUSSION}

The findings of our experiment have shown that they are relatively large and well-established networks do not perform very well malaria-infected function in the division of red blood cells, including ResNet50, DenseNet121, DPN92. These networks typically have a deep, complicated architecture and a huge number of parameters. They did not do as well, however, they're supposed to be worse than easy, personalized CNN models.

The number of parameters for personalized models they are just $5 \%$ or fewer, relative to those broad and well-established networks. This finding proves that there are two things, the first is the architecture of deep learning networks needs to be understood. The basic condition of the activities of classification. Styles that are performing well in the role of natural image recognition does not perform well for medical pictures. The second is the rise in Blindly, network complexity and parameters do not necessarily lead to better outcomes.

Specifically, in our task which is a binary classification of infected cells. The variability and complexity could not compare with and it is less than other tasks like a natural or medical image recognition task. Moreover, malaria-infected cell images usually have low recognition rates, large similarity, and blurred lesions areas. These reasons may affect the performance of models and lead the mentioned large, complex, and well-established networks did not work well. Therefore, it is important to use or design different models for different tasks that are simpler and more efficient in such tasks.

As a summary, based on Figure 5 and Figure, it is obvious that using the proposed customized $\mathrm{CNN}$ all the performance evaluation metrics (accuracy, specificity, sensitivity, and precision) when it is utilized using the statistical performance of it was $98.85 \%$ for accuracy, $98.90 \%$ for specificity, $98.79 \%$ for sensitivity and $98.90 \%$ for precision. As aforementioned, using the new CNN architecture with softmax classifier results are comparable with pre-trained CNNs using the same dataset the same classifier.

Table 4 shows that the proposed customer model beat all pre-trained $\mathrm{CNNs}$ in the time and required less time with a small difference (less than $0.5 \%$ ) in the performance evaluation metrics and with the smaller number of layers and by employing the smallest input size which means that this model is suitable to be used as a mobile application in the future. Finally, Figures 7 and 8 shows that the used model is very efficient on feature extractions and extract features from the regions of interest areas that models must pay attention to.

Table 4. Comparing the proposed architecture with other architectures in the literature ( ${ }^{*}$ Time Per Image)

\begin{tabular}{ccccccc}
\hline CNN Architecture & $\begin{array}{c}\text { Number } \\
\text { of Layers }\end{array}$ & $\begin{array}{c}\text { Input } \\
\text { Image Size }\end{array}$ & $\begin{array}{c}\text { Time Required for } \\
\text { Classification* }^{*}\end{array}$ & $\begin{array}{c}\text { Accuracy } \\
(\mathbf{\%})\end{array}$ & $\begin{array}{c}\text { Precision } \\
(\mathbf{\%})\end{array}$ & $\begin{array}{c}\text { F-Score } \\
(\%)\end{array}$ \\
\hline VGG-19 [4] & 47 & $224 \times 224$ & 0.2342 & 99.32 & 99.71 & 99.31 \\
SqueezeNet [4] & 68 & $227 \times 227$ & 0.3546 & 98.66 & 99.44 & 98.64 \\
InceptionResNet-V2 [4] & 825 & $229 \times 229$ & 3.8427 & 98.79 & 99.56 & 98.77 \\
AlexNet [3] & 25 & $227 \times 227$ & 0.1729 & 93.70 & -- & 93.7 \\
ResNet-50 [3] & 177 & $224 \times 224$ & 1.1517 & 95.7 & -- & 95.7 \\
Xception [3] & 171 & $229 \times 229$ & 1.0073 & 89.0 & -- & 89.5 \\
DenseNet-121 [3] & 709 & $224 \times 224$ & 3.0842 & 93.1 & -- & 93.1 \\
ADCN [18] & 23 & $100 \times 100$ & 1.1332 & 97.47 & -- & 97.5 \\
DCNN [19] & 32 & $128 \times 128$ & 2.2564 & 98.9 & -- & 98.70 \\
Proposed & 23 & $64 \times 64$ & 0.1352 & 98.85 & 98.90 & 98.85 \\
\hline
\end{tabular}

\section{CONCLUSIONS}

To improve the problem of the poor performance of traditional malaria detection methods, we propose a miniature and elegant novel CNN model named AOCTNet. In this paper, a customized convolutional neural network $(\mathrm{CNN})$ model for the detection of malaria using a segmented RBCs image dataset was proposed. The proposed architecture success in the detection of malaria with high performance in accuracy and sensitivity, the less time, and the smallest input size for the two classes when compared to pre-trained CNNs. This model provides high accuracy and effective classification that can be 
improved more by including more RBCs images with and without segmentation. Also, an improvement can be done for the proposed architecture and trying to optimize the hyperparameters using the genetic algorithm (GA) or any other optimization technique which can result in enhancing the training process. In the future, we hope that our proposed model can be extended to mobile devices using cloud technology, especially in areas with inadequate medical conditions and medical facilities. It would dramatically reduce the burden on doctors and improve the pace of diagnosis of malaria and decrease the damage caused by malaria.

\section{ACKNOWLEDGMENT}

The authors would thank Rajaraman et al. for sharing their dataset for public access. The authors would also thank the anonymous reviewers for their valuable comments.

\section{REFERENCES}

[1] World Health Organization. (2014). Malaria: fact sheet (No. WHO-EM/MAC/035/E). World Health Organization. Regional Office for the Eastern Mediterranean.

[2] Caraballo, H., King, K. (2014). Emergency department management of mosquito-borne illness: Malaria, dengue, and West Nile virus. Emergency Medicine Practice, 16(5): 1-23.

[3] Rajaraman, S., Antani, S.K., Poostchi, M., Silamut, K., Hossain, M.A., Maude, R.J., Jaeger, S., Thoma, G.R. (2018). Pre-trained convolutional neural networks as feature extractors toward improved malaria parasite detection in thin blood smear images. PeerJ, 6: e4568. https://doi.org/10.7717/peerj.4568

[4] Rajaraman, S., Jaeger, S., Antani, S.K. (2019). Performance evaluation of deep neural ensembles toward malaria parasite detection in thin-blood smear images. PeerJ, 7: e6977. https://doi.org/10.7717/peerj.6977

[5] Reddy, A.S.B., Juliet, D.S. (2019). Transfer learning with ResNet-50 for malaria cell-image classification. 2019 International Conference on Communication and Signal Processing (ICCSP), Chennai, India, pp. 09450949. https://doi.org/10.1109/ICCSP.2019.8697909

[6] Poostchi, M., Silamut, K., Maude, R.J., Jaeger, S., Thoma, G. (2018). Image analysis and machine learning for detecting malaria. Translational Research, 194: 36-55. https://doi.org/10.1016/j.trsl.2017.12.004

[7] Pan, W.D., Dong, Y., Wu, D. (2018). Classification of malaria-infected cells using deep convolutional neural networks. Machine Learning: Advanced Techniques and Emerging Applications, 159. https://doi.org/10.5772/intechopen.72426

[8] Alqudah, A.M., Alquraan, H., Qasmieh, I.A. (2019). Segmented and non-segmented skin lesions classification using transfer learning and adaptive moment learning rate technique using pretrained convolutional neural network. Journal of Biomimetics, Biomaterials and Biomedical Engineering, 42: 67-78.
https://doi.org/10.4028/www.scientific.net/JBBBE.42.6 7

[9] Vijayalakshmi, A., Rajesh Kanna, B (2019). Deep learning approach to detect malaria from microscopic images. Multimedia Tools and Applications, 79: 1529715317. https://doi.org/10.1007/s11042-019-7162-y

[10] Rahman, A., Zunair, H., Rahman, M.S., Yuki, J.Q., Biswas, S., Alam, M.A., Alam, N.B., Mahdy, M.R. (2019). Improving malaria parasite detection from red blood cell using deep convolutional neural networks. arXiv preprint arXiv:1907.10418. 2019 Jul 23.

[11] Alqudah, A.M. (2020). AOCT-NET: A convolutional network automated classification of multiclass retinal diseases using spectral-domain optical coherence tomography images. Medical \& Biological Engineering \& Computing, 58(1): 41-53. https://doi.org/10.1007/s11517-019-02066-y

[12] Alqudah, A.M. (2019). Towards classifying nonsegmented heart sound records using instantaneous frequency based features. Journal of Medical Engineering \& Technology, 43(7): 418-430. https://doi.org/10.1080/03091902.2019.1688408

[13] Alqudah, A.M., Algharib, H.M.S., Algharib, A.M.S., Algharib, H.M.S. (2019). Computer aided diagnosis system for automatic two stages classification of breast mass in digital mammogram images. Biomedical Engineering: Applications, Basis and Communications, 31(1): https://doi.org/10.4015/S1016237219500078

[14] Alqudah, A.M. (2019). Ovarian cancer classification using serum proteomic profiling and wavelet features a comparison of machine learning and features selection algorithms. Journal of Clinical Engineering, 44(4): 165173. https://doi.org/10.1097/JCE.0000000000000359

[15] Alqudah, A.M., Alquran, H., Qasmieh, I.A. (2020). Classification of heart sound short records using bispectrum analysis approach images and deep learning. Network Modeling Analysis in Health Informatics and Bioinformatics, $9(1)$ : 1-16. http://doi.org/10.1007/s13721-020-00272-5

[16] Alqudah, A., Qazan, S., Alquran, H., Qasmieh, I., Alqudah, A. (2020). COVID-19 detection from x-ray images using different artificial intelligence hybrid models. Jordan Journal of Electrical Engineering, 6(6): 168. http://doi.org/10.5455/jjee.204-1585312246

[17] Zhou, B., Khosla, A., Lapedriza, A., Oliva, A., Torralba, A. (2016). Learning deep features for discriminative localization. 2016 IEEE Conference on Computer Vision and Pattern Recognition (CVPR), Las Vegas, NV, pp. 2921-2929. https://doi.org/10.1109/CVPR.2016.319

[18] Quan, Q., Wang, J., Liu, L. (2020). An effective convolutional neural network for classifying red blood cells in malaria diseases. Interdisciplinary Sciences, Computational Life Sciences, 12: 217-225. https://doi.org/10.1007/s12539-020-00367-7

[19] Suriya, M., Chandran, V., Sumithra, M.G. (2019). Enhanced deep convolutional neural network for malarial parasite classification. International Journal of Computers and Applications, 1-10. https://doi.org/10.1080/1206212X.2019.1672277 